\title{
Global Synchronization Control of General Delayed Discrete-Time Networks With Stochastic Coupling and Disturbances
}

\author{
Jinling Liang, Zidong Wang, Senior Member, IEEE, Yurong Liu, and Xiaohui Liu
}

\begin{abstract}
In this paper, the synchronization control problem is considered for two coupled discrete-time complex networks with time delays. The network under investigation is quite general to reflect the reality, where the state delays are allowed to be time varying with given lower and upper bounds, and the stochastic disturbances are assumed to be Brownian motions that affect not only the network coupling but also the overall networks. By utilizing the Lyapunov functional method combined with linear matrix inequality (LMI) techniques, we obtain several sufficient delay-dependent conditions that ensure the coupled networks to be globally exponentially synchronized in the mean square. A control law is designed to synchronize the addressed coupled complex networks in terms of certain LMIs that can be readily solved using the Matlab LMI toolbox. Two numerical examples are presented to show the validity of our theoretical analysis results.
\end{abstract}

Index Terms-Control law, coupled networks, linear matrix inequality (LMI), Lyapunov functional, stochastic disturbance, synchronization, time-varying delay.

\section{INTRODUCTION}

C OMPLEX networks exist in our lives. For example, the brain is a neural network; the global economy is a network of national economies; computer viruses routinely spread through the Internet; food webs, ecosystems, and metabolic pathways can be represented by networks. The complexity of networks in the social, biological, engineering, and physical sciences gives rise to many challenges for scientists and engineers. In order to better understand the dynamical behaviors of different kinds of complex networks, an important and interesting phenomenon to investigate is the synchrony of all dynamical nodes. Synchronization problem have been attracting recurrent research interests for many complex networks that include, but are not limited to, large-scale and complex networks of chaotic oscillators [6], [12], [19], [27], the coupled systems exhibiting spatiotemporal chaos and autowaves [15], [26], and the array of coupled neural networks with or without delays [2], [8], [13], [14], [23]. For example, the theta rhythm related to the behavior of animals is produced by partial synchronization of neuronal

Manuscript received January 8, 2008; revised April 5, 2008. This work was supported by the Royal Society Sino-British Fellowship Trust Award of the U.K. This paper was recommended by Associate Editor J. Wang.

J. Liang is with the Department of Mathematics, Southeast University, Nanjing 210096, China (e-mail: jinlliang@gmail.com).

Z. Wang and X. Liu are with the Department of Information Systems and Computing, Brunel University, Uxbridge, UB8 3PH, U.K. (e-mail: Zidong. Wang@ brunel.ac.uk).

Y. Liu is with the Department of Mathematics, Yangzhou University, Yangzhou 225002, China (e-mail: liuyurong@ gmail.com).

Digital Object Identifier 10.1109/TSMCB.2008.925724 activity in the hippocampal network [22], and an excessive synchronization of the neuronal activity over a wide area in the brain results in the epileptic rhythm [17]. Moreover, it has recently been revealed that, for message delivery in networks, a good synchronization can help achieve secure communication in terms of stable and high transportation rate.

It has now been well recognized that time delays may cause undesirable dynamic network behaviors such as oscillation and instability [5], [10], [16], [24]. Therefore, synchronization problem for complex networks with time delays has gained increasing research attention. For example, the synchronization criteria have been established in [7] for complex dynamical network models with coupling delays for both continuous and discrete time cases, which have further been improved in [5] by using less conservative delay-dependent techniques. A variational method has been used in [11] to deal with the synchronization problem for an array of linearly coupled identical connected neural networks with delays, whereas the similar problem has been addressed in [23] for an array of coupled nonlinear systems with delay and nonreciprocal timevarying coupling. More recently, by using Lyapunov functional method and Kronecker product technique, the global exponential synchronization has been established in [3] for arrays of coupled identical delayed neural networks with constant and delayed coupling. A notable fact is that most of the existing results have been concerned with the synchronization problem for continuous-time and deterministic complex networks with or without delays.

As pointed out in [18], it is rather challenging to understand the interaction topology of complex networks because of the discrete and random nature of network topology. On one hand, in a real complex network, the signal transfer could be perturbed randomly from the release of probabilistic causes such as neurotransmitters [20] and packet dropouts [21]. Synchronization control problem for stochastic networks has recently begun to receive initial research attention. For example, in [25], the synchronization control problem has been considered for stochastic neural networks with time-varying delays, and a novel control method has been given to estimate the controller gain. In [9], the complete synchronization has been achieved between unidirectionally coupled Chua's circuits within stochastic perturbation. Furthermore, in [4], by introducing the stochastic coupling term, the complete synchronization problem has been investigated for an array of linearly stochastically coupled neural networks with time delays. It is worth mentioning that the network coupling could occur in both a deterministic and 
a stochastic way, and the stochastic perturbations could act on both the coupling term and the overall networks. On the other hand, discrete-time networks could be more suitable to model digitally transmitted signals in a dynamical way. Note that discrete-time networks have already been applied in a wide range of areas, such as image processing, time series analysis, quadratic optimization problems, and system identification. Unfortunately, despite its importance in practice, the global synchronization problem for discrete-time networks with both stochastic coupling and stochastic disturbances with or without delays has not been fully investigated yet, which constitutes the main focus of this paper.

In this paper, we are interested in the synchronization control problem for stochastic discrete-time complex networks with time delays, where the stochastic disturbances are assumed to be Brownian motions that affect not only the network coupling but also the overall networks. Our main purpose is to establish some delay-dependent criteria to ensure that the two identical delayed networks with stochastic disturbances are globally exponentially synchronized. Based on the Lyapunov functional method and the stochastic analysis theory, we like to analyze and design appropriate feedback controllers with the hope that the derived synchronization criteria can be expressed in the form of linear matrix inequalities (LMIs). Note that the LMIs can be effectively solved and checked by the algorithms such as the interior-point method [1].

The rest of this paper is organized as follows. In Section II, some notations are introduced first, and then the coupled network model is presented. In Section III, via the Lyapunov functional method combined with the LMI technique, main results for synchronization are obtained, and the controller design is proposed. Two illustrative examples are given in Section IV to demonstrate the effectiveness of the acquired results. Finally, in Section V, we give the conclusion of this paper.

\section{Notations AND PROBlem Formulation}

Throughout this paper, the notation $P>0$ means that $P$ is real symmetric and positive definite. $\|\cdot\|$ refers to the Euclidean vector norm and the induced matrix norm. $\lambda_{\min }(\cdot)$ and $\lambda_{\max }(\cdot)$ denote the minimum and maximum eigenvalue, respectively. In symmetric block matrices, we use an asterisk "*" to represent a term that is induced by symmetry. Let $\mathbb{E}\{\cdot\}$ be the mathematical expectation operator with respect to the given probability measure $\mathcal{P}$ and $(\Omega, \mathcal{F}, \mathcal{P})$ be a complete probability space with a natural filtration $\left\{\mathcal{F}_{t}\right\}_{t \geq 0}$. For integers $\alpha, \beta$ with $\alpha<\beta, \mathbb{N}[\alpha, \beta]$ denotes the discrete interval given by $\mathbb{N}[\alpha, \beta]=$ $\{\alpha, \alpha+1, \ldots, \beta-1, \beta\}$ and $C\left(\mathbb{N}[\alpha, \beta], \mathbb{R}^{n}\right)$ means the set of all functions $\phi: \mathbb{N}[\alpha, \beta] \rightarrow \mathbb{R}^{n}$. I stands for the identity matrix with appropriate dimensions. Matrices, if not explicitly stated, are assumed to have compatible dimensions.

To facilitate the readers, let us present the complex networks in a step-by-step way. We start with the following master network:

$$
s(k+1)=A s(k)+B f(s(k))+C f_{d}(s(k-\tau(k)))
$$

where $k=1,2, \ldots$ and $s(k)=\left(s_{1}(k), s_{2}(k), \ldots, s_{n}(k)\right)^{\mathrm{T}} \in \mathbb{R}^{n}$ is the state vector of the network; $A$ is a constant matrix; matrices $B$ and $C$ are the connection weight matrix and the delayed connection weight matrix, respectively; $\tau(k)$ is a time-varying delay in the state which satisfies

$$
\tau_{m} \leq \tau(k) \leq \tau_{M}
$$

where $\tau_{m}$ and $\tau_{M}$ are known positive integers representing the lower and upper bounds of the delay; $f(s(k))=$ $\left(f_{1}\left(s_{1}(k)\right), f_{2}\left(s_{2}(k)\right), \ldots, f_{n}\left(s_{n}(k)\right)\right)^{\mathrm{T}}$ and $f_{d}(s(k-\tau(k)))=$ $\left(f_{d 1}\left(s_{1}(k-\tau(k))\right), f_{d 2}\left(s_{2}(k-\tau(k))\right), \ldots, f_{d n}\left(s_{n}(k-\tau(k))\right)\right)^{\mathrm{T}}$ are the nonlinear functions. In system (1), $\{\phi(k): k=$ $\left.-\tau_{M},-\tau_{M}+1, \ldots, 0\right\}$ is a given initial condition sequence in $C\left(\mathbb{N}\left[-\tau_{M}, 0\right], \mathbb{R}^{n}\right)$.

Throughout of this paper, the following assumption is always made.

Assumption 1: [10] There exist constants $l_{i}^{-}, l_{i}^{+}, k_{i}^{-}, k_{i}^{+}$such that the following inequalities

$$
\begin{aligned}
l_{i}^{-} & \leq \frac{f_{i}(u)-f_{i}(v)}{u-v} \leq l_{i}^{+} \\
k_{i}^{-} & \leq \frac{f_{d i}(u)-f_{d i}(v)}{u-v} \leq k_{i}^{+}, \quad(i=1,2, \ldots, n)
\end{aligned}
$$

hold for all different $u, v \in \mathbb{R}$.

As discussed in the introduction, real-world networks are usually coupled, and stochastic disturbances could enter both the network coupling and the overall networks. Therefore, in this paper, an array of linearly coupled identical networks with time-varying delay under study (the slave networks) is proposed as follows (without loss of generality, we only consider the case that two networks are coupled):

$$
\begin{aligned}
x_{i}(k+1)= & A x_{i}(k)+B f\left(x_{i}(k)\right)+C f_{d}\left(x_{i}(k-\tau(k))\right)+u_{i}(k) \\
& +H\left(k, x_{i}(k)-s(k), x_{i}(k-\tau(k))-s(k-\tau(k))\right) \\
& \times \omega_{i 1}(k)+\sum_{j=1}^{2} G_{i j} \Gamma x_{j}(k)\left(d_{i}+\omega_{i 2}(k)\right)
\end{aligned}
$$

where $i=1,2$ and $x_{i}(k)=\left(x_{i 1}(k), x_{i 2}(k), \ldots, x_{i n}(k)\right)^{\mathrm{T}} \in$ $\mathbb{R}^{n} ; d_{i}>0$ denotes the coupling strength; $u_{i}(\cdot)$ is the control input to ensure that $x_{i}(k)-s(k) \rightarrow 0$ as $k \rightarrow \infty$; $\omega_{i 1}(\cdot), \omega_{i 2}(\cdot)(i=1,2)$ are independent scalar Wiener process (Brownian Motion) on the probability space $(\Omega, \mathcal{F}, \mathcal{P})$ satisfying

$$
\begin{aligned}
\mathbb{E}\left\{\omega_{i j}(k)\right\} & =0 \\
\mathbb{E}\left\{\omega_{i j}^{2}(k)\right\} & =1 \\
\mathbb{E}\left\{\omega_{i j}(s) \omega_{i j}(t)\right\} & =0(s \neq t)
\end{aligned}
$$

in which $i, j=1,2$. It is assumed that $H: \mathbb{R} \times \mathbb{R}^{n} \times \mathbb{R}^{n} \rightarrow$ $\mathbb{R}^{n}$ is the diffusion coefficient vector and there exist matrices $G_{1}, G_{2}$ such that

$$
\begin{aligned}
H^{\mathrm{T}}(k, x, y) H(k, x, y) \leq\left\|G_{1} x\right\|^{2}+\left\|G_{2} y\right\|^{2} & \\
\forall(k, x, y) & \in \mathbb{R} \times \mathbb{R}^{n} \times \mathbb{R}^{n} .
\end{aligned}
$$

Furthermore, $\Gamma \in \mathbb{R}^{n \times n}$ is a constant inner coupling matrix of the nodes, and $G=\left(G_{i j}\right)_{2 \times 2}$ is the out-coupling matrix of the 
network defined as follows. If there is a connection from node $j$ to node $i(i \neq j)$, then $G_{i j}>0$; otherwise, $G_{i j}=0$.

Remark 1: It can be seen that matrix $G$ reflects the topological structure of the networks and it also satisfies the diffusive coupling conditions

$$
G_{i i}=-\sum_{j \neq i} G_{i j}, \quad(i, j=1,2) .
$$

Moreover, (5) and (6) infer that when the synchronization is reached, the synchronization state is just a solution of an isolated node model (1).

Remark 2: It is notable that, in addition to the constant couplings in our model (3), we consider the state-dependent stochastic sequences $\omega_{i 1}(k)$ on the overall network and $\omega_{i 2}(k)$ on the coupling term. This represents one of the first attempts to deal with both deterministic and stochastic disturbances on the coupling as well as the overall network dynamics. In this sense, the model (3) is more natural and general than the existing ones including that introduced in [4].

In order to investigate the global synchronization for coupled networks (3), we let $e_{i}(k)=x_{i}(k)-s(k)$ be the synchronization error. Then, the error system follows immediately from (1) and (3) as follows:

$$
\begin{aligned}
e_{i}(k+1)= & A e_{i}(k)+B g\left(e_{i}(k)\right)+C g_{d}\left(e_{i}(k-\tau(k))\right) \\
& +u_{i}(k)+H\left(k, e_{i}(k), e_{i}(k-\tau(k))\right) \omega_{i 1}(k) \\
& +\sum_{j=1}^{2} G_{i j} \Gamma e_{j}(k)\left(d_{i}+\omega_{i 2}(k)\right)
\end{aligned}
$$

where $g\left(e_{i}(k)\right)=f\left(x_{i}(k)\right)-f(s(k))$ and $g_{d}\left(e_{i}(k-\tau(k))\right)=$ $f_{d}\left(x_{i}(k-\tau(k))\right)-f_{d}(s(k-\tau(k)))$.

From Assumption 1, one has

$$
\begin{array}{ll}
l_{i}^{-} \leq \frac{g_{i}(u)}{u} \leq l_{i}^{+}, \quad & k_{i}^{-} \leq \frac{g_{d i}(u)}{u} \leq k_{i}^{+}, \\
& (u \in \mathbb{R} \backslash\{0\}, \quad i=1,2, \ldots, n) .
\end{array}
$$

For simplicity, we denote $L_{1}=\operatorname{diag}\left\{l_{1}^{-} l_{1}^{+}, l_{2}^{-} l_{2}^{+}, \ldots, l_{n}^{-} l_{n}^{+}\right\}$, $L_{2}=\operatorname{diag}\left\{\left(-\left(l_{1}^{-}+l_{1}^{+}\right) / 2,-\left(l_{2}^{-}+l_{2}^{+}\right) / 2, \ldots,-\left(l_{n}^{-}+l_{n}^{+}\right) / 2\right\}\right.$; $K_{1}=\operatorname{diag}\left\{k_{1}^{-} k_{1}^{+}, k_{2}^{-} k_{2}^{+}, \ldots, k_{n}^{-} k_{n}^{+}\right\}, \quad K_{2}=\operatorname{diag}\left\{-\left(k_{1}^{-}+\right.\right.$ $\left.\left.k_{1}^{+}\right) / 2,-\left(k_{2}^{-}+k_{2}^{+}\right) / 2, \ldots,-\left(k_{n}^{-}+k_{n}^{+}\right) / 2\right\}$.

Definition 1: The coupled network system (3) is said to be globally exponentially synchronized in the mean square if there exist two constants $\vartheta>0$ and $\mu \in(0,1)$ such that

$$
\begin{aligned}
\mathbb{E}\left\{\left\|x_{i}(k)-s(k)\right\|^{2}\right\} & =\mathbb{E}\left\{\left\|e_{i}(k)\right\|^{2}\right\} \leq \vartheta \mu^{k} \\
& \times \max _{s \in \mathbb{N}\left[-\tau_{M}, 0\right]} \mathbb{E}\left\{\left\|e_{i}(s)\right\|^{2}\right\}, \quad i=1,2
\end{aligned}
$$

hold for all $k \geq \kappa$, where $\kappa$ is a sufficiently large positive integer.

To obtain our main results, we need the following lemma known as the Schur complement:

Lemma 1: Let $Q(x)=Q^{\mathrm{T}}(x), R(x)=R^{\mathrm{T}}(x)$, and $S(x)$ depend affinely on $x$ [1]. Then, the following LMI:

$$
\left[\begin{array}{cc}
Q(x) & S(x) \\
S^{\mathrm{T}}(x) & R(x)
\end{array}\right]>0
$$

holds if and only if one of the following conditions holds:

1) $R(x)>0, Q(x)-S(x) R^{-1}(x) S^{\mathrm{T}}(x)>0$;

2) $Q(x)>0, R(x)-S^{\mathrm{T}}(x) Q^{-1}(x) S(x)>0$.

\section{MAin Results}

In this section, by utilizing the Lyapunov functional method combining with the LMI techniques, let us first derive delaydependent stability criterion for the following unforced system of (7):

$$
\begin{aligned}
e_{i}(k+1)= & A e_{i}(k)+B g\left(e_{i}(k)\right)+C g_{d}\left(e_{i}(k-\tau(k))\right) \\
& +H\left(k, e_{i}(k), e_{i}(k-\tau(k))\right) \omega_{i 1}(k) \\
& +\sum_{j=1}^{2} G_{i j} \Gamma e_{j}(k)\left(d_{i}+\omega_{i 2}(k)\right)
\end{aligned}
$$

and then design a controller $u_{i}(k)$ that synchronizes the coupled network (3) with stochastic disturbances.

Theorem 1: The unforced system (9) is globally exponentially stable in the mean square if there exist seven positive definite matrices $P, Q_{1}, Q_{2}, R_{1}, R_{2}, \bar{R}_{1}, \bar{R}_{2}$, four positive diagonal matrices $S_{i}=\operatorname{diag}\left\{s_{i 1}, s_{i 2}, \ldots, s_{i n}\right\}(i=1,2,3,4)$ and a scalar $\lambda>0$ such that the following LMIs hold:

$$
P<\lambda I \quad \Xi=\left[\begin{array}{ll}
\Xi_{11} & \Xi_{12} \\
\Xi_{12}^{\mathrm{T}} & \Xi_{22}
\end{array}\right]<0
$$

where we have the expressions for $\Xi_{11}, \Xi_{12}$, and $\Xi_{22}$, shown at the bottom of the next page, with $\Pi_{2}=\left(G_{11} G_{12}\left(1+d_{1}^{2}\right)+\right.$ $\left.G_{22} G_{21}\left(1+d_{2}^{2}\right)\right) \Gamma^{T} P \Gamma+d_{1} G_{12} A^{T} P \Gamma+d_{2} G_{21} \Gamma^{T} P A$

$$
\begin{aligned}
\Pi_{1}= & \left(\tau_{M}-\tau_{m}+1\right) Q_{1}+A^{\mathrm{T}} P A-P+\lambda G_{1}^{\mathrm{T}} G_{1}-S_{1} L_{1} \\
& +R_{1}+\bar{R}_{1}+\left(\left(1+d_{1}^{2}\right) G_{11}^{2}+\left(1+d_{2}^{2}\right) G_{21}^{2}\right) \Gamma^{\mathrm{T}} P \Gamma \\
& +d_{1} G_{11}\left(A^{\mathrm{T}} P \Gamma+\Gamma^{\mathrm{T}} P A\right) \\
\Pi_{3}= & \left(\tau_{M}-\tau_{m}+1\right) Q_{2}+A^{\mathrm{T}} P A-P+\lambda G_{1}^{\mathrm{T}} G_{1}-S_{2} L_{1} \\
& +R_{2}+\bar{R}_{2}+\left(\left(1+d_{1}^{2}\right) G_{12}^{2}+\left(1+d_{2}^{2}\right) G_{22}^{2}\right) \Gamma^{\mathrm{T}} P \Gamma \\
& +d_{2} G_{22}\left(A^{\mathrm{T}} P \Gamma+\Gamma^{\mathrm{T}} P A\right) .
\end{aligned}
$$

Proof: It follows easily from (8) that

$$
\begin{array}{r}
\left(g_{i}\left(e_{1 i}(k)\right)-l_{i}^{-} e_{1 i}(k)\right)\left(g_{i}\left(e_{1 i}(k)\right)-l_{i}^{+} e_{1 i}(k)\right) \leq 0, \\
i=1,2, \ldots, n
\end{array}
$$

which is equivalent to

$$
\begin{aligned}
& {\left[\begin{array}{c}
e_{1}(k) \\
g\left(e_{1}(k)\right)
\end{array}\right]\left[\begin{array}{cc}
l_{i}^{-} l_{i}^{+} \delta_{i} \delta_{i}^{\mathrm{T}} & -\frac{l_{i}^{-}+l_{i}^{+}}{2} \delta_{i} \delta_{i}^{\mathrm{T}} \\
-\frac{l_{i}^{-}+l_{i}^{+}}{2} \delta_{i} \delta_{i}^{\mathrm{T}} & \delta_{i} \delta_{i}^{\mathrm{T}}
\end{array}\right]} \\
& \quad \times\left[\begin{array}{lll}
e_{1}^{\mathrm{T}}(k) & \left.g^{\mathrm{T}}\left(e_{1}(k)\right)\right] \leq 0, \quad i=1,2, \ldots, n
\end{array}\right.
\end{aligned}
$$

where $\delta_{i}$ is the $n$-dimensional unit column vector having one element on its $i$ th row and zeros elsewhere. Multiplying both sides of (12) by $s_{1 i}$ and summing up from 1 to $n$ with respect to $i$, we have

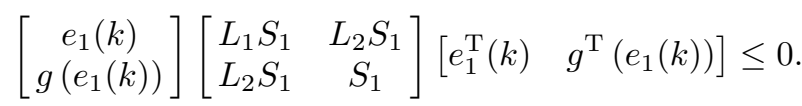


Similarly, we have the following inequalities:

$$
\begin{aligned}
& {\left[\begin{array}{c}
e_{2}(k) \\
g\left(e_{2}(k)\right)
\end{array}\right]\left[\begin{array}{cc}
L_{1} S_{2} & L_{2} S_{2} \\
L_{2} S_{2} & S_{2}
\end{array}\right]\left[\begin{array}{cc}
e_{2}^{\mathrm{T}}(k) & \left.g^{\mathrm{T}}\left(e_{2}(k)\right)\right] \leq 0 \\
{\left[\begin{array}{c}
e_{1}(k-\tau(k)) \\
g_{d}\left(e_{1}(k-\tau(k))\right)
\end{array}\right]\left[\begin{array}{cc}
K_{1} S_{3} & K_{2} S_{3} \\
K_{2} S_{3} & S_{3}
\end{array}\right]} \\
\quad \times\left[e_{1}^{\mathrm{T}}(k-\tau(k)) g_{d}^{\mathrm{T}}\left(e_{1}(k-\tau(k))\right)\right] \leq 0 \\
{\left[\begin{array}{c}
e_{2}(k-\tau(k)) \\
g_{d}\left(e_{2}(k-\tau(k))\right)
\end{array}\right]\left[\begin{array}{cc}
K_{1} S_{4} & K_{2} S_{4} \\
K_{2} S_{4} & S_{4}
\end{array}\right]} \\
\quad \times\left[e_{2}^{\mathrm{T}}(k-\tau(k))\right. & \left.g_{d}^{\mathrm{T}}\left(e_{2}(k-\tau(k))\right)\right] \leq 0
\end{array}\right.}
\end{aligned}
$$

Consider the following Lyapunov functional of system (9):

$$
\begin{aligned}
V(k)= & V_{1}(k)+V_{2}(k)+V_{3}(k)+V_{4}(k)+V_{5}(k) \\
= & \sum_{i=1}^{2} e_{i}^{\mathrm{T}}(k) P e_{i}(k)+\sum_{i=1}^{2} \sum_{j=k-\tau(k)}^{k-1} e_{i}^{\mathrm{T}}(j) Q_{i} e_{i}(j) \\
& +\sum_{i=1}^{2} \sum_{j=k-\tau_{m}}^{k-1} e_{i}^{\mathrm{T}}(j) R_{i} e_{i}(j)+\sum_{i=1}^{2} \sum_{j=k-\tau_{M}}^{k-1} e_{i}^{\mathrm{T}}(j) \bar{R}_{i} e_{i}(j) \\
& +\sum_{i=1}^{2} \sum_{j=-\tau_{M}}^{-\tau_{m}} \sum_{m=k+j}^{k-1} e_{i}^{\mathrm{T}}(m) Q_{i} e_{i}(m) .
\end{aligned}
$$

Calculating the difference of $V_{1}(k)$ along the solutions of (9) and taking the mathematical expectation, noting the independent properties of stochastic processes $\omega_{i 1}(\cdot), \omega_{i 2}(\cdot)$, and (4), we obtain (18), shown at the bottom of the next page.

Similarly, we have

$$
\begin{aligned}
\mathbb{E}\left\{\Delta V_{2}(k)\right\}=\sum_{i=1}^{2} \mathbb{E}\{ & e_{i}^{\mathrm{T}}(k) Q_{i} e_{i}(k)-e_{i}^{\mathrm{T}}(k-\tau(k)) \\
& \times Q_{i} e_{i}(k-\tau(k)) \\
& +\sum_{j=k+1-\tau(k+1)}^{k-\tau_{m}} e_{i}^{\mathrm{T}}(j) Q_{i} e_{i}(j)
\end{aligned}
$$

$$
\begin{aligned}
& +\sum_{j=k-\tau_{m}+1}^{k-1} e_{i}^{\mathrm{T}}(j) Q_{i} e_{i}(j) \\
& \left.-\sum_{j=k-\tau(k)+1}^{k-1} e_{i}^{\mathrm{T}}(j) Q_{i} e_{i}(j)\right\} \\
& \leq \sum_{i=1}^{2} \mathbb{E}\left\{e_{i}^{\mathrm{T}}(k) Q_{i} e_{i}(k)-e_{i}^{\mathrm{T}}(k-\tau(k))\right. \\
& \times Q_{i} e_{i}(k-\tau(k)) \\
& \left.+\sum_{j=k+1-\tau_{M}}^{k-\tau_{m}} e_{i}^{\mathrm{T}}(j) Q_{i} e_{i}(j)\right\} \\
& \mathbb{E}\left\{\Delta V_{3}(k)\right\}=\sum_{i=1}^{2} \mathbb{E}\left\{e_{i}^{\mathrm{T}}(k) R_{i} e_{i}(k)-e_{i}^{\mathrm{T}}\left(k-\tau_{m}\right)\right. \\
& \left.\times R_{i} e_{i}\left(k-\tau_{m}\right)\right\} \\
& \mathbb{E}\left\{\Delta V_{4}(k)\right\}=\sum_{i=1}^{2} \mathbb{E}\left\{e_{i}^{\mathrm{T}}(k) \bar{R}_{i} e_{i}(k)-e_{i}^{\mathrm{T}}\left(k-\tau_{M}\right)\right. \\
& \left.\times \bar{R}_{i} e_{i}\left(k-\tau_{M}\right)\right\} \\
& \mathbb{E}\left\{\Delta V_{5}(k)\right\}=\sum_{i=1}^{2} \mathbb{E}\left\{\left(\tau_{M}-\tau_{m}\right) e_{i}^{\mathrm{T}}(k) Q_{i} e_{i}(k)\right. \\
& \left.-\sum_{j=k+1-\tau_{M}}^{k-\tau_{m}} e_{i}^{\mathrm{T}}(j) Q_{i} e_{i}(j)\right\} .
\end{aligned}
$$

Conditions (5) and (10) indicate that

$$
\begin{aligned}
& H^{\mathrm{T}}\left(k, e_{i}(k), e_{i}(k-\tau(k))\right) P H\left(k, e_{i}(k), e_{i}(k-\tau(k))\right) \\
& \leq \lambda\left(e_{i}^{\mathrm{T}}(k) G_{1}^{\mathrm{T}} G_{1} e_{i}(k)+e_{i}^{\mathrm{T}}(k-\tau(k)) G_{2}^{\mathrm{T}} G_{2} e_{i}(k-\tau(k))\right), \\
& i=1,2 .
\end{aligned}
$$

$$
\begin{aligned}
& \Xi_{11}=\left[\begin{array}{cccccc}
\Pi_{1} & 0 & 0 & 0 & A^{\mathrm{T}} P B-S_{1} L_{2}+d_{1} G_{11} \Gamma^{\mathrm{T}} P B & A^{\mathrm{T}} P C+d_{1} G_{11} \Gamma^{\mathrm{T}} P C \\
* & -R_{1} & 0 & 0 & 0 & 0 \\
* & * & -Q_{1}+\lambda G_{2}^{\mathrm{T}} G_{2}-S_{3} K_{1} & 0 & 0 & -S_{3} K_{2} \\
* & * & * & -\bar{R}_{1} & 0 & 0 \\
* & * & * & * & B^{\mathrm{T}} P B-S_{1} & B^{\mathrm{T}} P C \\
* & * & * & * & * & C^{\mathrm{T}} P C-S_{3}
\end{array}\right] \\
& \Xi_{12}=\left[\begin{array}{cccccc}
\Pi_{2} & 0 & 0 & 0 & d_{2} G_{21} \Gamma^{\mathrm{T}} P B & d_{2} G_{21} \Gamma^{\mathrm{T}} P C \\
0 & 0 & 0 & 0 & 0 & 0 \\
0 & 0 & 0 & 0 & 0 & 0 \\
0 & 0 & 0 & 0 & 0 & 0 \\
d_{1} G_{12} B^{\mathrm{T}} P \Gamma & 0 & 0 & 0 & 0 & 0 \\
d_{1} G_{12} C^{\mathrm{T}} P \Gamma & 0 & 0 & 0 & 0 & 0
\end{array}\right] \\
& \Xi_{22}=\left[\begin{array}{cccccc}
\Pi_{3} & 0 & 0 & 0 & A^{\mathrm{T}} P B-S_{2} L_{2}+d_{2} G_{22} \Gamma^{\mathrm{T}} P B & A^{\mathrm{T}} P C+d_{2} G_{22} \Gamma^{\mathrm{T}} P C \\
* & -R_{2} & 0 & 0 & 0 & 0 \\
* & * & -Q_{2}+\lambda G_{2}^{\mathrm{T}} G_{2}-S_{4} K_{1} & 0 & 0 & -S_{4} K_{2} \\
* & * & * & -\bar{R}_{2} & 0 & 0 \\
* & * & * & * & B^{\mathrm{T}} P B-S_{2} & B^{\mathrm{T}} P C \\
* & * & * & * & * & C^{\mathrm{T}} P C-S_{4}
\end{array}\right]
\end{aligned}
$$


Denoting

$$
\begin{gathered}
\xi(k)=\left(e_{1}^{\mathrm{T}}(k) e_{1}^{\mathrm{T}}\left(k-\tau_{m}\right) e_{1}^{\mathrm{T}}(k-\tau(k)) e_{1}^{\mathrm{T}}\left(k-\tau_{M}\right)\right. \\
g^{\mathrm{T}}\left(e_{1}(k)\right) g_{d}^{\mathrm{T}}\left(e_{1}(k-\tau(k))\right) e_{2}^{\mathrm{T}}(k) \\
e_{2}^{\mathrm{T}}\left(k-\tau_{m}\right) e_{2}^{\mathrm{T}}(k-\tau(k)) e_{2}^{\mathrm{T}}\left(k-\tau_{M}\right) \\
\left.g^{\mathrm{T}}\left(e_{2}(k)\right) g_{d}^{\mathrm{T}}\left(e_{2}(k-\tau(k))\right)\right)^{\mathrm{T}}
\end{gathered}
$$

it follows from (13)-(16) and (18)-(23) that

$\mathbb{E}\{\Delta V(k)\} \leq \mathbb{E}\left\{\xi^{\mathrm{T}}(k) \Xi \xi(k)\right\}$

$$
\leq-\lambda_{\min }(-\Xi) \mathbb{E}\left\{\left\|e_{1}(k)\right\|^{2}+\left\|e_{2}(k)\right\|^{2}\right\} \text {. }
$$

From the definition (17) of $V(k)$, it is easy to obtain that

$$
\begin{aligned}
& \mathbb{E}\{V(k)\} \geq \lambda_{\min }(P) \mathbb{E}\left\{\left\|e_{1}(k)\right\|^{2}+\left\|e_{2}(k)\right\|^{2}\right\} \\
& \mathbb{E}\{V(k)\} \leq \lambda_{\max }(P) \mathbb{E}\left\{\left\|e_{1}(k)\right\|^{2}+\left\|e_{2}(k)\right\|^{2}\right\} \\
&+\rho \mathbb{E}\left\{\sum_{j=k-\tau_{M}}^{k-1}\left(\left\|e_{1}(j)\right\|^{2}+\left\|e_{2}(j)\right\|^{2}\right)\right\}
\end{aligned}
$$

where $\rho=\left(\tau_{M}-\tau_{m}+1\right) \max \left\{\lambda_{\max }\left(Q_{1}\right), \lambda_{\max }\left(Q_{2}\right)\right\}+\max$ $\left\{\lambda_{\max }\left(R_{1}\right), \lambda_{\max }\left(R_{2}\right)\right\}+\max \left\{\lambda_{\max }\left(\bar{R}_{1}\right), \lambda_{\max }\left(\bar{R}_{2}\right)\right\}$. For any given scalar $\varepsilon>1$, taking $k$ to be sufficiently large, we have from (24) and (26) that

$$
\begin{aligned}
& \mathbb{E}\left\{\varepsilon^{k} V(k)\right\}=\mathbb{E}\left\{V(0)+\sum_{j=0}^{k-1}\left(\varepsilon^{j+1} \Delta V(j)+\varepsilon^{j}(\varepsilon-1) V(j)\right)\right\} \\
& \leq \mathbb{E}\left\{\lambda_{\max }(P)\left(\left\|e_{1}(0)\right\|^{2}+\left\|e_{2}(0)\right\|^{2}\right)\right. \\
&+\rho \sum_{j=-\tau_{M}}^{-1}\left(\left\|e_{1}(j)\right\|^{2}+\left\|e_{2}(j)\right\|^{2}\right) \\
&+\left(-\varepsilon \lambda_{\min }(-\Xi)+(\varepsilon-1) \lambda_{\max }(P)\right) \\
& \\
& \times \sum_{j=0}^{k-1} \varepsilon^{j}\left(\left\|e_{1}(j)\right\|^{2}+\left\|e_{2}(j)\right\|^{2}\right)+(\varepsilon-1) \rho \\
&\left.\times \sum_{j=0}^{k-1} \sum_{l=j-\tau_{M}}^{j-1} \varepsilon^{j}\left(\left\|e_{1}(l)\right\|^{2}+\left\|e_{2}(l)\right\|^{2}\right)\right\}
\end{aligned}
$$

$\mathbb{E}\left\{\Delta V_{1}(k)\right\}=\mathbb{E}\left\{V_{1}(k+1)-V_{1}(k)\right\}$

$$
\begin{aligned}
& =\sum_{i=1}^{2} \mathbb{E}\left\{\left[A e_{i}(k)+B g\left(e_{i}(k)\right)+C g_{d}\left(e_{i}(k-\tau(k))\right)+d_{i} \sum_{j=1}^{2} G_{i j} \Gamma e_{j}(k)\right]^{\mathrm{T}}\right. \\
& \times P\left[A e_{i}(k)+B g\left(e_{i}(k)\right)+C g_{d}\left(e_{i}(k-\tau(k))\right)+d_{i} \sum_{j=1}^{2} G_{i j} \Gamma e_{j}(k)\right] \\
& -e_{i}^{\mathrm{T}}(k) P e_{i}(k)+H\left(k, e_{i}(k), e_{i}(k-\tau(k))\right) P H\left(k, e_{i}(k), e_{i}(k-\tau(k))\right) \\
& \left.+\left(\sum_{j=1}^{2} G_{i j} \Gamma e_{j}(k)\right)^{\mathrm{T}} P\left(\sum_{j=1}^{2} G_{i j} \Gamma e_{j}(k)\right)\right\} \\
& =\sum_{i=1}^{2} \mathbb{E}\left\{e_{i}^{\mathrm{T}}(k)\left(A^{\mathrm{T}} P A-P\right) e_{i}(k)+g_{d}^{\mathrm{T}}\left(e_{i}(k-\tau(k))\right) C^{\mathrm{T}} P C g_{d}\left(e_{i}(k-\tau(k))\right)\right. \\
& +g^{\mathrm{T}}\left(e_{i}(k)\right) B^{\mathrm{T}} P B g\left(e_{i}(k)\right)+2 e_{i}^{\mathrm{T}}(k) A^{\mathrm{T}} P\left[B g\left(e_{i}(k)\right)+C g_{d}\left(e_{i}(k-\tau(k))\right)\right] \\
& \left.+2 g^{\mathrm{T}}\left(e_{i}(k)\right) B^{\mathrm{T}} P C g_{d}\left(e_{i}(k-\tau(k))\right)+H\left(k, e_{i}(k), e_{i}(k-\tau(k))\right) P H\left(k, e_{i}(k), e_{i}(k-\tau(k))\right)\right\} \\
& +\mathbb{E}\left\{\left(\left(1+d_{1}^{2}\right) G_{11}^{2}+\left(1+d_{2}^{2}\right) G_{21}^{2}\right) e_{1}^{\mathrm{T}}(k) \Gamma^{\mathrm{T}} P \Gamma e_{1}(k)+\left(\left(1+d_{1}^{2}\right) G_{12}^{2}+\left(1+d_{2}^{2}\right) G_{22}^{2}\right)\right. \\
& \times e_{2}^{\mathrm{T}}(k) \Gamma^{\mathrm{T}} P \Gamma e_{2}(k)+2\left(G_{11} G_{12}\left(1+d_{1}^{2}\right)+G_{21} G_{22}\left(1+d_{2}^{2}\right)\right) e_{1}^{\mathrm{T}}(k) \Gamma^{\mathrm{T}} P \Gamma e_{2}(k) \\
& +2\left[d_{1} G_{11} e_{1}^{\mathrm{T}}(k) A^{\mathrm{T}} P \Gamma e_{1}(k)+e_{1}^{\mathrm{T}}(k)\left(d_{1} G_{12} A^{\mathrm{T}} P \Gamma+d_{2} G_{21} \Gamma^{\mathrm{T}} P A\right) e_{2}(k)+d_{2} G_{22} e_{2}^{\mathrm{T}}(k) A^{\mathrm{T}} P \Gamma e_{2}(k)\right] \\
& +2\left[d_{1} G_{11} e_{1}^{\mathrm{T}}(k) \Gamma^{\mathrm{T}} P B g\left(e_{1}(k)\right)+d_{1} G_{12} e_{2}^{\mathrm{T}}(k) \Gamma^{\mathrm{T}} P B g\left(e_{1}(k)\right)\right. \\
& \left.+d_{2} G_{21} e_{1}^{\mathrm{T}}(k) \Gamma^{\mathrm{T}} P B g\left(e_{2}(k)\right)+d_{2} G_{22} e_{2}^{\mathrm{T}}(k) \Gamma^{\mathrm{T}} P B g\left(e_{2}(k)\right)\right] \\
& +2\left[d_{1} G_{11} e_{1}^{\mathrm{T}}(k) \Gamma^{\mathrm{T}} P C g_{d}\left(e_{1}(k-\tau(k))\right)+d_{1} G_{12} e_{2}^{\mathrm{T}}(k) \Gamma^{\mathrm{T}} P C g_{d}\left(e_{1}(k-\tau(k))\right)\right. \\
& \left.\left.+d_{2} G_{21} e_{1}^{\mathrm{T}}(k) \Gamma^{\mathrm{T}} P C g_{d}\left(e_{2}(k-\tau(k))\right)+d_{2} G_{22} e_{2}^{\mathrm{T}}(k) \Gamma^{\mathrm{T}} P C g_{d}\left(e_{2}(k-\tau(k))\right)\right]\right\}
\end{aligned}
$$


where

$$
\begin{gathered}
\mathbb{E}\left\{\sum_{j=0}^{k-1} \sum_{l=j-\tau_{M}}^{j-1} \varepsilon^{j}\left(\left\|e_{1}(l)\right\|^{2}+\left\|e_{2}(l)\right\|^{2}\right)\right\} \\
\leq \mathbb{E}\left\{\tau_{M} \varepsilon^{\tau_{M}} \sum_{l=-\tau_{M}}^{-1}\left(\left\|e_{1}(l)\right\|^{2}+\left\|e_{2}(l)\right\|^{2}\right)\right. \\
+\left(\sum_{l=0}^{k-1-\tau_{M}} \sum_{s=1}^{\tau_{M}}+\sum_{l=k-\tau_{M}}^{k-1} \sum_{s=1}^{k-l}\right) \\
\left.\quad \times \varepsilon^{l} \varepsilon^{s}\left(\left\|e_{1}(l)\right\|^{2}+\left\|e_{2}(l)\right\|^{2}\right)\right\} \\
\leq \tau_{M}^{2} \varepsilon^{\tau_{M}} \sup _{l \in \mathbb{N}\left[-\tau_{M}, 0\right]} \mathbb{E}\left\{\left\|e_{1}(l)\right\|^{2}+\left\|e_{2}(l)\right\|^{2}\right\} \\
+\tau_{M} \varepsilon^{\tau_{M}} \mathbb{E}\left\{\sum_{l=0}^{k-1} \varepsilon^{l}\left(\left\|e_{1}(l)\right\|^{2}+\left\|e_{2}(l)\right\|^{2}\right)\right\} .
\end{gathered}
$$

Substituting (28) into (27) gives

$$
\begin{aligned}
\mathbb{E}\left\{\varepsilon^{k} V(k)\right\} & \leq p_{1}(\varepsilon) \sup _{l \in \mathbb{N}\left[-\tau_{M}, 0\right]} \mathbb{E}\left\{\left\|e_{1}(l)\right\|^{2}+\left\|e_{2}(l)\right\|^{2}\right\} \\
& +p_{2}(\varepsilon) \mathbb{E}\left\{\sum_{l=0}^{k} \varepsilon^{l}\left(\left\|e_{1}(l)\right\|^{2}+\left\|e_{2}(l)\right\|^{2}\right)\right\}
\end{aligned}
$$

where $p_{1}(\varepsilon)=\lambda_{\max }(P)+\rho \tau_{M}+\rho(\varepsilon-1) \tau_{M}^{2} \varepsilon^{\tau_{M}}, p_{2}(\varepsilon)=$ $(\varepsilon-1) \lambda_{\max }(P)-\varepsilon \lambda_{\min }(-\Xi)+\rho(\varepsilon-1) \tau_{M} \varepsilon^{\tau_{M}}$. Since $p_{1}(\varepsilon)$ and $p_{2}(\varepsilon)$ are continuous functions of $\varepsilon$ and $p_{1}(1)>0, p_{2}(1)<$ 0 , there must exist a scalar $\mu>1$ such that $p_{1}(\mu)>0$ and $p_{2}(\mu) \leq 0$, which leads to the fact that

$$
\begin{aligned}
\mathbb{E}\left\{\left\|e_{1}(k)\right\|^{2}+\left\|e_{2}(k)\right\|^{2}\right\} & \leq \frac{p_{1}(\mu)}{\lambda_{\min }(P)}\left(\frac{1}{\mu}\right)^{k} \sup _{l \in \mathbb{N}\left[-\tau_{M}, 0\right]} \\
& \times \mathbb{E}\left\{\left\|e_{1}(l)\right\|^{2}+\left\|e_{2}(l)\right\|^{2}\right\} .
\end{aligned}
$$

From Definition 1, (30) means that the coupled network system (9) is globally exponentially stable in the mean square, and the proof is then completed.

Next, we are going to design a controller $u_{i}(k)$ in order to make the coupled system (3) to be synchronized. For simplicity of the implementation, we adopt the following memoryless state-feedback controller:

$$
u_{i}(k)=K e_{i}(k) .
$$

Substitute (31) into (7) to give the following closed-loop system:

$$
\begin{aligned}
e_{i}(k+1)= & (A+K) e_{i}(k)+B g\left(e_{i}(k)\right) \\
& +C g_{d}\left(e_{i}(k-\tau(k))\right) \\
& +H\left(k, e_{i}(k), e_{i}(k-\tau(k))\right) \omega_{i 1}(k) \\
& +\sum_{j=1}^{2} G_{i j} \Gamma e_{j}(k)\left(d_{i}+\omega_{i 2}(k)\right)
\end{aligned}
$$

where $K \in \mathbb{R}^{n \times n}$ is a constant gain matrix to be determined.
Theorem 2: The coupled stochastic disturbed system (3) is globally exponentially synchronized in the mean square via the memoryless state-feedback controller (31) if there exist seven positive definite matrices $P, Q_{1}, Q_{2}, R_{1}, R_{2}, \bar{R}_{1}, \bar{R}_{2}$, four positive diagonal matrices $S_{i}(i=1,2,3,4)$, one arbitrary matrix $\widetilde{K}$, and a scalar $\lambda>0$ such that the following LMIs hold:

$$
P<\lambda I \quad \Sigma=\left[\begin{array}{cc}
\Sigma_{11} & \Sigma_{12} \\
\Sigma_{12}^{\mathrm{T}} & \Sigma_{22}
\end{array}\right]<0
$$

where the expressions for $\Sigma_{11}, \Sigma_{12}, \Sigma_{22}, \Upsilon_{2}, \Upsilon_{1}$, and $\Upsilon_{3}$ are shown at the bottom of the next page. Moreover, the controller gain is given by $K=P^{-1} \widetilde{K}^{\mathrm{T}}$.

Proof: From Theorem 1, one knows that system (33) is globally exponentially stable in the mean square if there exist seven matrices $P>0, Q_{1}>0, Q_{2}>0, R_{1}>0, R_{2}>0$, $\bar{R}_{1}>0, \bar{R}_{2}>0$, four diagonal matrices $S_{i}>0(i=1,2,3,4)$, and a scalar $\lambda>0$ such that

$$
P<\lambda I \quad \widetilde{\Xi}=\left[\begin{array}{cc}
\widetilde{\Xi}_{11} & \widetilde{\Xi}_{12} \\
\widetilde{\Xi}_{12}^{\mathrm{T}} & \widetilde{\Xi}_{22}
\end{array}\right]<0
$$

where $\widetilde{\Xi}_{11}, \widetilde{\Xi}_{12}$, and $\widetilde{\Xi}_{22}$ are similar as $\Xi_{11}, \Xi_{12}$, and $\Xi_{22}$ in Theorem 1 with the only difference that $A$ is substituted by $A+K$.

On the other hand, by Lemma $1, \widetilde{\Xi}<0$ is equivalent to

$$
\widetilde{\Sigma}=\left[\begin{array}{ll}
\widetilde{\Sigma}_{11} & \widetilde{\Sigma}_{12} \\
\widetilde{\Sigma}_{12}^{\mathrm{T}} & \widetilde{\Sigma}_{22}
\end{array}\right]<0
$$

where $\widetilde{\Sigma}_{11}, \widetilde{\Sigma}_{12}$, and $\widetilde{\Sigma}_{22}$ are the same as $\Sigma_{11}, \Sigma_{12}$, and $\Sigma_{22}$ by just noting that $K=P^{-1} \widetilde{K}^{\mathrm{T}}$.

In the following, one special case is discussed. The proof of the subsequent corollary is similar to that of Theorem 2 and, hence, omitted here. Consider the coupled network system (3) without stochastic terms. In this case, (3) reduces to

$$
\begin{aligned}
& x_{i}(k+1)=A x_{i}(k)+B f\left(x_{i}(k)\right) \\
& \quad+C f_{d}\left(x_{i}(k-\tau(k))\right)+u_{i}(k)+d_{i} \sum_{j=1}^{2} G_{i j} \Gamma x_{j}(k)
\end{aligned}
$$

and we can obtain the following result:

Corollary 1: The discrete-time coupled system (36) is globally exponentially synchronized via the memoryless statefeedback controller (31) if there exist seven positive definite matrices $P, Q_{1}, Q_{2}, R_{1}, R_{2}, \bar{R}_{1}, \bar{R}_{2}$, four positive diagonal matrices $S_{i}(i=1,2,3,4)$ and one arbitrary matrix $\widetilde{K}$ such that the following LMI holds:

$$
\Delta=\left[\begin{array}{ll}
\Delta_{11} & \Delta_{12} \\
\Delta_{12}^{\mathrm{T}} & \Delta_{22}
\end{array}\right]<0
$$

where the expressions for $\Delta_{11}, \Delta_{12}, \Delta_{22}, \Theta_{2}, \Theta_{1}$, and $\Theta_{3}$ are shown at the bottom of page 1080. Moreover, the controller gain $K=P^{-1} \widetilde{K}^{\mathrm{T}}$.

Remark 3: In our main results, the synchronization control problem is considered for two coupled discrete-time complex networks with time-delays, and the control law is designed to synchronize the addressed coupled complex networks in 
terms of certain LMIs that can be readily solved using Matlab LMI toolbox. It should be pointed out that the variables of the LMIs are essentially the parameters of the addressed complex networks. Therefore, once an adequate complex network is established, and the corresponding parameters are identified, we can analyze the exponential synchronization control problem of the complex network by simply checking the feasibility of the LMIs. Note that the verification of the solvability of LMIs can be conveniently done by utilizing the numerically efficient Matlab LMI toolbox, and no turning of parameters will be needed [1]. In the past decade, LMIs have gained much attention for their computational tractability and usefulness in system engineering (see, e.g., [1]) as the so-called interior point method has been proved to be numerically very efficient for solving the LMIs. The number of analysis and design problems that can be formulated as LMI problems is large and continues to grow.

\section{Two Numerical Examples}

In this section, two simple examples are presented to justify Theorem 2 acquired in the previous section.
Example 1: Consider an isolated network (1) with parameters as follows:

$$
\begin{aligned}
\tau_{m} & =2 \\
A & =\left[\begin{array}{cccc}
-0.0830 & -0.9386 & -0.6559 & -0.5539 \\
-0.6616 & -0.5905 & -0.4519 & -0.6801 \\
-0.5170 & -0.4406 & -0.8397 & -0.3672 \\
-0.1710 & -0.9419 & -0.5326 & -0.2393
\end{array}\right] \\
B & =\left[\begin{array}{cccc}
-0.002 & 0.01 & 0.001 & 0 \\
0 & -0.002 & -0.013 & 0.01 \\
-0.01 & 0.001 & 0.002 & 0 \\
0 & 0 & 0.01 & 0.012
\end{array}\right] \\
C & =\left[\begin{array}{cccc}
0.03 & 0 & 0 & 0.03 \\
-0.03 & 0.03 & 0 & 0.03 \\
0 & -0.03 & 0.003 & 0.03 \\
0 & 0.03 & -0.03 & 0.036
\end{array}\right]
\end{aligned}
$$

and $\quad f(s(k))=f_{d}(s(k))=\left(\tanh \left(2 s_{1}(k)\right), \tanh \left(-4 s_{2}(k)\right)\right.$, $\left.\tanh \left(-2 s_{1}(k)\right), \tanh \left(2 s_{1}(k)\right)\right)^{\mathrm{T}}$. It can be easily determined that $K_{1}=L_{1}=0$ and $K_{2}=L_{2}=\operatorname{diag}\{-1,2,1,-1\}$.

$$
\begin{aligned}
& \Sigma_{11}=\left[\begin{array}{ccccccc}
\Upsilon_{1} & 0 & 0 & 0 & -S_{1} L_{2}+d_{1} G_{11} \Gamma^{\mathrm{T}} P B & d_{1} G_{11} \Gamma^{\mathrm{T}} P C & A^{\mathrm{T}} P+\widetilde{K} \\
* & -R_{1} & 0 & 0 & 0 & 0 & 0 \\
* & * & -Q_{1}+\lambda G_{2}^{\mathrm{T}} G_{2}-S_{3} K_{1} & 0 & 0 & -S_{3} K_{2} & 0 \\
* & * & * & -\bar{R}_{1} & 0 & 0 & 0 \\
* & * & * & * & -S_{1} & 0 & B^{\mathrm{T}} P \\
* & * & * & * & * & -S_{3} & C^{\mathrm{T}} P \\
* & * & * & * & * & * & -P
\end{array}\right] \\
& \Sigma_{12}=\left[\begin{array}{ccccccc}
\Upsilon_{2} & 0 & 0 & 0 & d_{2} G_{21} \Gamma^{\mathrm{T}} P B & d_{2} G_{21} \Gamma^{\mathrm{T}} P C & 0 \\
0 & 0 & 0 & 0 & 0 & 0 & 0 \\
0 & 0 & 0 & 0 & 0 & 0 & 0 \\
0 & 0 & 0 & 0 & 0 & 0 & 0 \\
d_{1} G_{12} B^{\mathrm{T}} P \Gamma & 0 & 0 & 0 & 0 & 0 & 0 \\
d_{1} G_{12} C^{\mathrm{T}} P \Gamma & 0 & 0 & 0 & 0 & 0 & 0 \\
0 & 0 & 0 & 0 & 0 & 0 & 0
\end{array}\right] \\
& \Sigma_{22}=\left[\begin{array}{ccccccc}
\Upsilon_{3} & 0 & 0 & 0 & -S_{2} L_{2}+d_{2} G_{22} \Gamma^{\mathrm{T}} P B & d_{2} G_{22} \Gamma^{\mathrm{T}} P C & A^{\mathrm{T}} P+\widetilde{K} \\
* & -R_{2} & 0 & 0 & 0 & 0 & 0 \\
* & * & -Q_{2}+\lambda G_{2}^{\mathrm{T}} G_{2}-S_{4} K_{1} & 0 & 0 & -S_{4} K_{2} & 0 \\
* & * & * & -\bar{R}_{2} & 0 & 0 & 0 \\
* & * & * & * & -S_{2} & 0 & B^{\mathrm{T}} P \\
* & * & * & * & * & -S_{4} & C^{\mathrm{T}} P \\
* & * & * & * & * & * & -P
\end{array}\right] \\
& \Upsilon_{2}=\left(G_{11} G_{12}\left(1+d_{1}^{2}\right)+G_{22} G_{21}\left(1+d_{2}^{2}\right)\right) \Gamma^{\mathrm{T}} P \Gamma+d_{1} G_{12}\left(A^{\mathrm{T}} P \Gamma+\tilde{K} \Gamma\right)+d_{2} G_{21}\left(\Gamma^{\mathrm{T}} P A+\Gamma^{\mathrm{T}} \tilde{K}^{\mathrm{T}}\right) \\
& \Upsilon_{1}=\left(\tau_{M}-\tau_{m}+1\right) Q_{1}-P+\lambda G_{1}^{\mathrm{T}} G_{1}-S_{1} L_{1}+R_{1}+\bar{R}_{1}+\left(\left(1+d_{1}^{2}\right) G_{11}^{2}+\left(1+d_{2}^{2}\right) G_{21}^{2}\right) \Gamma^{\mathrm{T}} P \Gamma \\
& +d_{1} G_{11}\left(A^{\mathrm{T}} P \Gamma+\Gamma^{\mathrm{T}} P A+\widetilde{K} \Gamma+\Gamma^{\mathrm{T}} \widetilde{K}^{\mathrm{T}}\right) \\
& \Upsilon_{3}=\left(\tau_{M}-\tau_{m}+1\right) Q_{2}-P+\lambda G_{1}^{\mathrm{T}} G_{1}-S_{2} L_{1}+R_{2}+\bar{R}_{2}+\left(\left(1+d_{1}^{2}\right) G_{12}^{2}+\left(1+d_{2}^{2}\right) G_{22}^{2}\right) \Gamma^{\mathrm{T}} P \Gamma \\
& +d_{2} G_{22}\left(A^{\mathrm{T}} P \Gamma+\Gamma^{\mathrm{T}} P A+\widetilde{K} \Gamma+\Gamma^{\mathrm{T}} \widetilde{K}^{\mathrm{T}}\right)
\end{aligned}
$$


Now, let two identical networks in (1) be coupled and disturbed with the following parameters:

$$
\begin{aligned}
d_{1} & =0.1, \quad d_{2}=0.2 \\
G & =\left[\begin{array}{cccc}
-0.1-a & 0.1+a \\
0.2 & -0.2
\end{array}\right] \\
\Gamma & =\left[\begin{array}{cccc}
-0.0579 & -0.0444 & -0.0404 & -0.0260 \\
-0.0867 & -0.0300 & -0.0390 & -0.0087 \\
-0.0407 & -0.0401 & -0.0360 & -0.0429 \\
-0.0113 & -0.0833 & -0.0140 & -0.0257
\end{array}\right] \\
G_{1} & =\left[\begin{array}{llll}
0.0595 & 0.0990 & 0.1570 & 0.0007 \\
0.0850 & 0.1413 & 0.0148 & 0.0441 \\
0.0238 & 0.0487 & 0.0788 & 0.0003
\end{array}\right] \\
G_{2} & =\left[\begin{array}{llll}
0.0568 & 0.0525 & 0.2703 & 0.1448 \\
0.0427 & 0.0416 & 0.2818 & 0.1128 \\
0.0804 & 0.1797 & 0.0664 & 0.1571
\end{array}\right] .
\end{aligned}
$$

Let $a=0$ and $\tau_{M}$ vary from the value 3. By using the Matlab LMI Control Toolbox, a feasible solution to the LMIs in (33) can always be found when $\tau_{M} \in \mathbb{N}[3,5]$. For example, taking $\tau_{M}=4$, the corresponding solution is listed as follows:

$$
P=\left[\begin{array}{cccc}
122.7081 & 5.7527 & 6.1273 & 4.8039 \\
5.7527 & 131.0024 & 7.1094 & 6.4317 \\
6.1273 & 7.1094 & 141.2300 & 16.6209 \\
4.8039 & 6.4317 & 16.6209 & 124.8004
\end{array}\right]
$$

$$
\begin{aligned}
Q_{1}= & {\left[\begin{array}{cccc}
18.8309 & 2.2670 & 3.8593 & 3.0423 \\
2.2670 & 21.1439 & 4.3440 & 4.3243 \\
3.8593 & 4.3440 & 32.4577 & 10.2498 \\
3.0423 & 4.3243 & 10.2498 & 23.0821
\end{array}\right] } \\
Q_{2}= & {\left[\begin{array}{cccc}
18.8315 & 2.2673 & 3.8595 & 3.0424 \\
2.2673 & 21.1442 & 4.3441 & 4.3243 \\
3.8595 & 4.3441 & 32.4578 & 10.2500 \\
3.0424 & 4.3243 & 10.2500 & 23.0829
\end{array}\right] }
\end{aligned}
$$$$
R_{1}=\bar{R}_{1}=\left[\begin{array}{cccc}
18.6853 & -1.4547 & -2.6639 & -1.7570 \\
-1.4547 & 16.9616 & -3.3628 & -2.6644 \\
-2.6639 & -3.3628 & 11.7259 & -5.0486 \\
-1.7570 & -2.6644 & -5.0486 & 16.4176
\end{array}\right]
$$$$
R_{2}=\bar{R}_{2}=\left[\begin{array}{cccc}
18.6854 & -1.4543 & -2.6635 & -1.7567 \\
-1.4543 & 16.9614 & -3.3624 & -2.6640 \\
-2.6635 & -3.3624 & 11.7260 & -5.0485 \\
-1.7567 & -2.6640 & -5.0485 & 16.4171
\end{array}\right]
$$$$
\widetilde{K}=\left[\begin{array}{cccc}
18.1433 & 91.8042 & 82.3544 & 34.6644 \\
128.1228 & 91.4326 & 88.0246 & 133.0618 \\
90.7563 & 70.6068 & 134.8563 & 87.5989 \\
75.0734 & 94.9697 & 63.6401 & 41.3365
\end{array}\right]
$$

$S_{1}=\operatorname{diag}\{13.0810,4.0177,7.3422,10.3274\}$

$S_{2}=\operatorname{diag}\{13.0815,4.0183,7.3428,10.3272\}$

$S_{3}=\operatorname{diag}\{8.5475,1.9206,3.1295,5.6770\}$

$S_{4}=\operatorname{diag}\{8.5480,1.9208,3.1298,5.6775\}$

$\lambda=159.7163$.

$\Delta_{11}=\left[\begin{array}{ccccccc}\Theta_{1} & 0 & 0 & 0 & -S_{1} L_{2}+d_{1} G_{11} \Gamma^{\mathrm{T}} P B & d_{1} G_{11} \Gamma^{\mathrm{T}} P C & A^{\mathrm{T}} P+\widetilde{K} \\ * & -R_{1} & 0 & 0 & 0 & 0 & 0 \\ * & * & -Q_{1}-S_{3} K_{1} & 0 & 0 & -S_{3} K_{2} & 0 \\ * & * & * & -\bar{R}_{1} & 0 & 0 & 0 \\ * & * & * & * & -S_{1} & 0 & B^{\mathrm{T}} P \\ * & * & * & * & * & -S_{3} & C^{\mathrm{T}} P \\ * & * & * & * & * & * & -P\end{array}\right]$

$\Delta_{12}=\left[\begin{array}{ccccccc}\Theta_{2} & 0 & 0 & 0 & d_{2} G_{21} \Gamma^{\mathrm{T}} P B & d_{2} G_{21} \Gamma^{\mathrm{T}} P C & 0 \\ 0 & 0 & 0 & 0 & 0 & 0 & 0 \\ 0 & 0 & 0 & 0 & 0 & 0 & 0 \\ 0 & 0 & 0 & 0 & 0 & 0 & 0 \\ d_{1} G_{12} B^{\mathrm{T}} P \Gamma & 0 & 0 & 0 & 0 & 0 & 0 \\ d_{1} G_{12} C^{\mathrm{T}} P \Gamma & 0 & 0 & 0 & 0 & 0 & 0 \\ 0 & 0 & 0 & 0 & 0 & 0 & 0\end{array}\right]$

$\Delta_{22}=\left[\begin{array}{ccccccc}\Theta_{3} & 0 & 0 & 0 & -S_{2} L_{2}+d_{2} G_{22} \Gamma^{\mathrm{T}} P B & d_{2} G_{22} \Gamma^{\mathrm{T}} P C & A^{\mathrm{T}} P+\widetilde{K} \\ * & -R_{2} & 0 & 0 & 0 & 0 & 0 \\ * & * & -Q_{2}-S_{4} K_{1} & 0 & 0 & -S_{4} K_{2} & 0 \\ * & * & * & -\bar{R}_{2} & 0 & 0 & 0 \\ * & * & * & * & -S_{2} & 0 & B^{\mathrm{T}} P \\ * & * & * & * & * & -S_{4} & C^{\mathrm{T}} P \\ * & * & * & * & * & * & -P\end{array}\right]$

$\Theta_{2}=\left(G_{11} G_{12} d_{1}^{2}+G_{22} G_{21} d_{2}^{2}\right) \Gamma^{\mathrm{T}} P \Gamma+d_{1} G_{12}\left(A^{\mathrm{T}} P \Gamma+\widetilde{K} \Gamma\right)+d_{2} G_{21}\left(\Gamma^{\mathrm{T}} P A+\Gamma^{\mathrm{T}} \widetilde{K}^{\mathrm{T}}\right)$

$\Theta_{1}=\left(\tau_{M}-\tau_{m}+1\right) Q_{1}-P-S_{1} L_{1}+R_{1}+\bar{R}_{1}+\left(d_{1}^{2} G_{11}^{2}+d_{2}^{2} G_{21}^{2}\right) \Gamma^{\mathrm{T}} P \Gamma+d_{1} G_{11}\left(A^{\mathrm{T}} P \Gamma+\Gamma^{\mathrm{T}} P A+\widetilde{K} \Gamma+\Gamma^{\mathrm{T}} \widetilde{K}^{\mathrm{T}}\right)$

$\Theta_{3}=\left(\tau_{M}-\tau_{m}+1\right) Q_{2}-P-S_{2} L_{1}+R_{2}+\bar{R}_{2}+\left(d_{1}^{2} G_{12}^{2}+d_{2}^{2} G_{22}^{2}\right) \Gamma^{\mathrm{T}} P \Gamma+d_{2} G_{22}\left(A^{\mathrm{T}} P \Gamma+\Gamma^{\mathrm{T}} P A+\widetilde{K} \Gamma+\Gamma^{\mathrm{T}} \widetilde{K}^{\mathrm{T}}\right)$ 
Moreover, the controller gain matrix is obtained by

$$
K=P^{-1} \widetilde{K}^{\mathrm{T}}=\left[\begin{array}{llll}
0.0840 & 0.9578 & 0.6559 & 0.5533 \\
0.6602 & 0.5858 & 0.4379 & 0.6696 \\
0.5262 & 0.4416 & 0.8406 & 0.3662 \\
0.1704 & 0.9403 & 0.5421 & 0.2266
\end{array}\right] \text {. }
$$

From Theorem 2, we know that the two coupled subsystems with stochastic disturbances and different initial conditions are globally exponentially synchronized with the given control law. Now, to show that the value of the parameter " $a$ " does influence the synchronous motion, we let $\tau_{M} \equiv 4$, and the parameter " $a$ " vary from -0.1 . According to our main results, the LMIs in (33) are always feasible when $a \in[-0.1,2.4853]$. In other words, the coupled network is synchronized when $a \in[-0.1,2.4853]$.

Example 2: In order to analyze the influence of stochastic disturbances onto the dynamics of the coupled system, we now consider model (36) without stochastic disturbances and then compare the synchronous behavior with that in Example 1. The coefficients and parameters are the same as those in Example 1. By using the Matlab LMI Control Toolbox, a solution to the LMI in (37) can always be found when $\tau_{M} \in \mathbb{N}[3,53]$. Comparing to the results in Example 1, we arrive at the conclusion that the random disturbances reduce the synchronous dynamics. For example, take $\tau_{M}=53$, the corresponding solution is listed as follows:

$$
\begin{aligned}
& P=\left[\begin{array}{cccc}
470.8045 & 153.3697 & -41.7123 & -106.7561 \\
153.3697 & 823.3083 & -24.4258 & -337.0709 \\
-41.7123 & -24.4258 & 143.3909 & 59.6581 \\
-106.7561 & -337.0709 & 59.6581 & 425.6428
\end{array}\right] \\
& Q_{1}=\left[\begin{array}{cccc}
7.4625 & 2.9786 & -0.0726 & -1.5957 \\
2.9786 & 15.3541 & -0.5364 & -6.2125 \\
-0.0726 & -0.5364 & 1.9650 & 0.7852 \\
-1.5957 & -6.2125 & 0.7852 & 7.5622
\end{array}\right] \\
& Q_{2}=\left[\begin{array}{cccc}
7.4905 & 2.9875 & -0.0617 & -1.5946 \\
2.9875 & 15.3753 & -0.5344 & -6.2285 \\
-0.0617 & -0.5344 & 1.9718 & 0.7872 \\
-1.5946 & -6.2285 & 0.7872 & 7.5860
\end{array}\right] \\
& \widetilde{K}=\left[\begin{array}{cccc}
100.2649 & 485.3352 & 66.0326 & -127.1442 \\
421.8741 & 301.5249 & 65.1491 & 126.7220 \\
282.9604 & 257.8321 & 114.8793 & 63.3447 \\
323.7366 & 551.0384 & 26.5753 & -166.5562
\end{array}\right] \\
& R_{1}=\bar{R}_{1}=\left[\begin{array}{cccc}
26.2741 & -0.9600 & -14.2561 & -8.7078 \\
-0.9600 & 7.9973 & 1.2198 & -5.5421 \\
-14.2561 & 1.2198 & 12.1709 & 6.8628 \\
-8.7078 & -5.5421 & 6.8628 & 10.1536
\end{array}\right] \\
& R_{2}=\bar{R}_{2}=\left[\begin{array}{cccc}
26.2832 & -0.8757 & -14.1574 & -8.6561 \\
-0.8757 & 7.7390 & 1.3061 & -5.1645 \\
-14.1574 & 1.3061 & 12.2651 & 6.8822 \\
-8.6561 & -5.1645 & 6.8822 & 9.7521
\end{array}\right] \\
& S_{1}=\operatorname{diag}\{9.4119,0.9675,8.0734,5.5794\} \\
& S_{2}=\operatorname{diag}\{9.7495,0.9816,7.8801,5.4895\} \\
& S_{3}=\operatorname{diag}\{4.8229,1.2810,0.8603,2.3303\} \\
& S_{4}=\operatorname{diag}\{4.8486,1.2796,0.8589,2.3449\} \text {. }
\end{aligned}
$$

Moreover, the controller gain matrix is obtained as

$$
K=\left[\begin{array}{llll}
0.0835 & 0.9575 & 0.6558 & 0.5532 \\
0.6594 & 0.5857 & 0.4380 & 0.6699 \\
0.5261 & 0.4416 & 0.8409 & 0.3661 \\
0.1707 & 0.9398 & 0.5423 & 0.2266
\end{array}\right]
$$

From Corollary 1, we know that the two coupled subsystems without stochastic disturbances and with different initial conditions are globally exponentially synchronized with the given control law.

\section{CONCLUSION}

In this paper, the synchronization problem has been analyzed for two identical coupled discrete-time complex networks with time-varying delay. In the complex system, both the overall networks and the network couplings are subject to stochastic disturbances. First, an easy-to-verify condition has been established under which the synchronization error dynamics is globally exponentially stable in the mean square. Second, a controller is designed to guarantee the coupled system to be synchronized by using a combination of LMI approach and the stochastic analysis tools. The LMI-based conditions obtained in this paper are dependent not only on the lower bound but also on the upper bound of the time-varying delay, which can be solved efficiently via the Matlab LMI Toolbox. Two numerical examples have been presented to show the validity of our theoretical analysis results.

\section{REFERENCES}

[1] S. Boyd, L. E. Ghaoui, E. Feron, and V. Balakrishnan, Linear Matrix Inequalities in System and Control Theory. Philadelphia, PA: SIAM, 1994

[2] J. Cao and J. Lu, "Adaptive synchronization of neural networks with or without time-varying delays," Chaos, vol. 16, no. 1, p. 013133 , Mar. 2006.

[3] J. Cao, P. Li, and W. W. Wang, "Global synchronization in arrays of delayed neural networks with constant and delayed coupling," Phys. Lett. $A$, vol. 353, no. 4, pp. 318-325, 2006.

[4] J. Cao, Z. Wang, and Y. Sun, "Synchronization in an array of linearly stochastically coupled neural networks with time delays," Phys. A, vol. 385, no. 2, pp. 718-728, 2007.

[5] H. Gao, J. Lam, and G. Chen, "New criteria for synchronization stability of general complex dynamical networks with coupling delays," Phys. Lett. A, vol. 360, no. 2, pp. 263-273, 2006.

[6] J. Jost and M. Joy, "Special properties and synchronization in coupled map lattices," Phys. Rev. E, Stat. Phys. Plasmas Fluids Relat. Interdiscip. Top., vol. 65, no. 1, p. 061 201, 2002.

[7] C. G. Li and G. Chen, "Synchronization in general complex dynamical networks with coupling delays," Phys. A, vol. 343, pp. 263-278, 2004.

[8] Z. Li and G. Chen, "Global synchronization and asymptotic stability of complex dynamical networks," IEEE Trans. Circuits Syst. II, Exp. Briefs, vol. 53, no. 1, pp. 28-33, Jan. 2006.

[9] W. Lin and Y. He, "Complete synchronization of the noise-perturbed Chua's circuits," Chaos, vol. 15, no. 2, p. 023 705, 2005.

[10] Y. Liu, Z. Wang, A. Serrano, and X. Liu, "Discrete-time recurrent neural networks with time-varying delays: Exponential stability analysis," Phys. Lett. A, vol. 362, no. 5/6, pp. 480-488, 2007.

[11] W. L. Lu and T. P. Chen, "Synchronization of coupled connected neura networks with delays," IEEE Trans. Circuits Syst. I, Reg. Papers, vol. 51, no. 12 , pp. 2491-2503, Dec. 2004

[12] W. L. Lu, T. P. Chen, and G. Chen, "Synchronization analysis of linearly coupled systems described by differential equations with a coupling delay," Phys. D-Nonlinear Phenom., vol. 221, no. 2, pp. 118-134, 2006. 
[13] J. H. Lü and G. Chen, "A time-varying complex dynamical network model and its controlled synchronization criteria," IEEE Trans. Autom. Control, vol. 50, no. 6, pp. 841-846, Jun. 2005.

[14] L. M. Pecora and T. L. Carroll, "Synchronization in chaotic systems," Phys. Rev. Lett., vol. 64, no. 8, pp. 821-824, 1990.

[15] V. Perez-Munuzuri, V. Perez-Villar, and L. O. Chua, "Autowaves for image processing on a two-dimensional CNN array of excitable nonlinear circuits: Flat and wrinkled labyrinths," IEEE Trans. Circuits Syst. I, Fundam. Theory Appl., vol. 40, no. 3, pp. 174-181, Mar. 1993.

[16] Q. Song and J. Cao, "Impulsive effects on stability of fuzzy CohenGrossberg neural networks with time-varying delays," IEEE Trans. Syst., Man, Cybern. B, Cybern., vol. 37, no. 3, pp. 733-741, Jun. 2007.

[17] K. Tateno, H. Hayashi, and S. Ishizuka, "Complexity of spatiotemporal activity of a neural network model which depends on the degree of synchronization," Neural Netw., vol. 11, no. 6, pp. 985-1003, Aug. 1998.

[18] Z. Toroczkai, "Complex networks: The challenge of interaction topology," Los Alamos Sci., vol. 29, pp. 94-109, 2005.

[19] X.-F. Wang and G. Chen, "Synchronization in small-world dynamical networks,"Int. J. Bifurc. Chaos, vol. 12, no. 1, pp. 187-192, 2002.

[20] Z. Wang, Y. Liu, M. Li, and X. Liu, "Stability analysis for stochastic Cohen-Grossberg neural networks with mixed time delays," IEEE Trans. Neural Netw., vol. 17, no. 3, pp. 814-820, May 2006.

[21] Z. Wang, F. Yang, D. W. C. Ho, and X. Liu, "Robust $H_{\infty}$ control for networked systems with random packet losses," IEEE Trans. Syst., Man, Cybern. B, Cybern., vol. 37, no. 4, pp. 916-924, Aug. 2007.

[22] J. Winson, "Patterns of hippocampal theta rhythm in the freely moving rat," Electroencephalogr. Clin. Neurophysiol., vol. 36, no. 3, pp. 291-301, Mar. 1974.

[23] C. W. Wu, "Synchronization in arrays of coupled nonlinear systems with delay and nonreciprocal time-varying coupling," IEEE Trans. Circuits Syst. II, Exp. Briefs, vol. 52, no. 5, pp. 282-286, May 2005.

[24] K. Yuan, J. Cao, and H.-X. Li, "Robust stability of switched CohenGrossberg neural networks with mixed time-varying delays," IEEE Trans. Syst., Man, Cybern. B, Cybern., vol. 36, no. 6, pp. 1356-1363, Dec. 2006.

[25] W. W. Yu and J. Cao, "Synchronization control of stochastic delayed neural networks," Phys. A, vol. 373, pp. 252-260, 2007.

[26] A. Zheleznyak and L. O. Chua, "Coexistence of low- and highdimensional spatio-temporal chaos in a chain of dissipatively coupled Chua's circuits," Int. J. Bifurc. Chaos, vol. 4, no. 3, pp. 639-674, 1994.

[27] J. Zhou and T. P. Chen, "Synchronization in general complex delayed dynamical networks," IEEE Trans. Circuits Syst. I, Reg. Papers, vol. 53, no. 3, pp. 733-744, Mar. 2006.

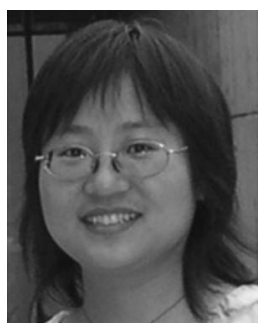

Jinling Liang received the B.Sc. and M.Sc. degrees in mathematics from Northwest University, Xi'an, China, in 1997 and 1999, respectively, and the Ph.D. degree in applied mathematics from Southeast University, Nanjing, China, in 2006.

She is currently an Associate Professor with the Department of Mathematics, Southeast University, Nanjing. From January 2004 to March 2004, she was a Research Assistant with the University of Hong Kong. From March to April 2004, she was a Research Assistant with the City University of Hong Kong. From April 2007 to March 2008, she was a Postdoctoral Research Fellow with the Department of Information Systems and Computing, Brunel University, Uxbridge, U.K., sponsored by the Royal Society Sino-British Fellowship Trust Award of the U.K. Her research interests include neural networks, complex networks, nonlinear systems, and bioinformatics. She has published around 20 papers in refereed international journals.

Dr. Liang is a member of program committee for several international conferences and serves as a very active Reviewer for many international journals.

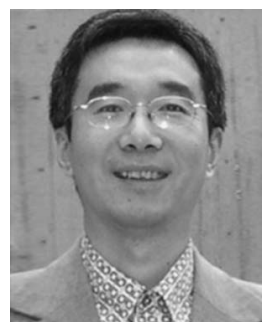

Zidong Wang (M'03-SM'03) was born in Jiangsu, China, in 1966. He received the B.Sc. degree in mathematics from Suzhou University, Suzhou, China, in 1986, and the M.Sc. degree in applied mathematics and the Ph.D. degree in electrical and computer engineering from the Nanjing University of Science and Technology, Nanjing, China, in 1990 and 1994, respectively.

$\mathrm{He}$ is currently a Professor of dynamical systems and computing with Brunel University, Uxbridge, U.K. He was appointed as a Lecturer in 1990 and Associate Professor in 1994 with the Nanjing University of Science and Technology. From January 1997 to December 1998, he was an Alexander von Humboldt Research Fellow with the Control Engineering Laboratory, Ruhr-University Bochum, Bochum, Germany. From January 1999 to February 2001, he was a Lecturer with the Department of Mathematics, University of Kaiserslautern, Kaiserslautern, Germany. From March 2001 to July 2002, he was a University Senior Research Fellow with the School of Mathematical and Information Sciences, Coventry University, U.K. In August 2002, he was with the Department of Information Systems and Computing, Brunel University, U.K., as a Lecturer, and was promoted to Reader in September 2003 and to Professor in July 2007. He is also holding an Adjunct TePin Professorship at Donghua University, Shanghai, China, a Visiting Professorship at Fuzhou University, Fuzhou, China, and a Guest Professorship at Nanjing Normal University, Nanjing. His research interests include dynamical systems, signal processing, bioinformatics, control theory, and applications. He has published more than 80 papers in refereed international journals.

Dr. Wang is a Fellow of the Royal Statistical Society, a member of program committees for many international conferences, and a very active Reviewer for many international journals. He was nominated an Appreciated Reviewer for the IEEE Transactions on Signal Processing in 2006 and 2007, an Outstanding Reviewer for the IEEE TRANSACTIONS ON Automatic CONTROL in 2004 and for the journal Automatica in 2000. He received the Standing Membership of the Technical Committee on Control of the International Association of Science and Technology for Development in 2000. From 2001 to 2007, he served as a member of technical program committees for 35 international conferences in the general area of systems theory and computing. He was awarded the Humboldt research fellowship in 1996 from the Alexander von Humboldt Foundation, the JSPS Research Fellowship in 1998 from the Japan Society for the Promotion of Science, and the William Mong Visiting Research Fellowship in 2002 from the University of Hong Kong. He was a recipient of the Outstanding Science and Technology Development Awards, once in 2005 and twice in 1997 from the National Education Committee of China, once in 1997 from the Military Industry General Company of China, once in 1997 from Jiangsu Province Government of China, and once in 1999 from the National Education Ministry of China. $\mathrm{He}$ is currently serving as an Associate Editor for the IEEE TRANSACTIONS on Automatic Control, IEEE Transactions on NeUral Networks, IEEE TRAnsactions on Signal Processing, IEEE Transactions on Systems, MAN, AND CYBERNETICS-PART C, IEEE TRANSACTIONS ON Control Systems Technology, and Circuits, Systems and Signal Processing, an Action Editor for Neural Networks, an Editorial Board Member for the International Journal of Systems Science, Neurocomputing, International Journal of Computer Mathematics, International Journal of General Systems, and an Associate Editor on the Conference Editorial Board for the IEEE Control Systems Society.

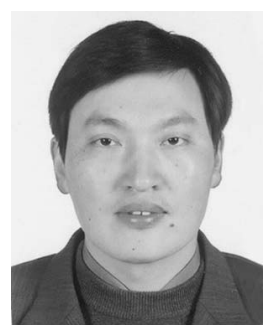

Yurong Liu received the B.Sc. degree in mathematics from Suzhou University, Suzhou, China, in 1986, the M.Sc. degree in applied mathematics from the Nanjing University of Science and Technology, Nanjing, China, in 1989, and the Ph.D. degree in applied mathematics from Suzhou University in 2000.

$\mathrm{He}$ is currently a Professor with the Department of Mathematics, Yangzhou University, Yangzhou, China. His current interests include neural networks, nonlinear dynamics, time-delay systems, and chaotic dynamics. 


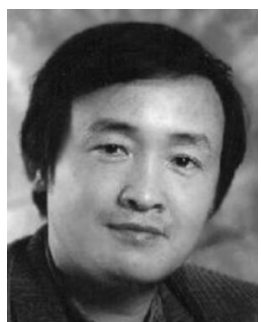

Xiaohui Liu received the B.Eng. degree in computing from Hohai University, Nanjing, China, in 1982 and the Ph.D. degree in computer science from Heriot-Watt University, Edinburg, U.K., in 1988.

$\mathrm{He}$ is currently a Professor of computing with Brunel University, Uxbridge, U.K. He leads the Intelligent Data Analysis (IDA) Group, performing interdisciplinary research involving artificial intelligence, dynamic systems, image and signal processing, and statistics, particularly for applications in biology, engineering, and medicine.

Prof. Liu serves on editorial boards of four computing journals, founded the biennial international conference series on IDA in 1995, and has given numerous invited talks in bioinformatics, data mining, and statistics conferences. 